Inertial/Magnetic Sensors based Pedestrian Dead Reckoning by Means of Multi-sensor Fusion

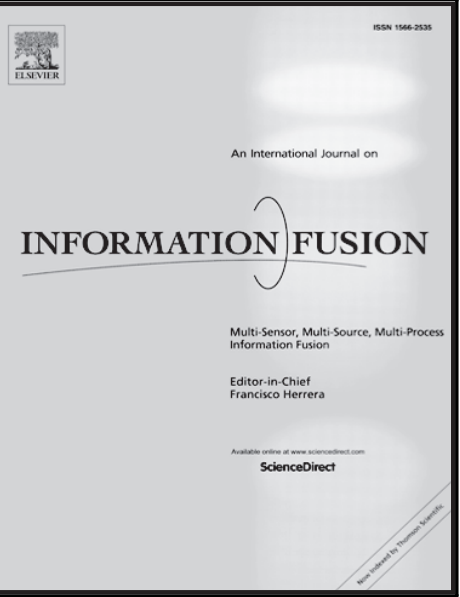

Sen Qiu, Zhelong Wang, Hongyu Zhao, Kairong Qin, Zhenglin Li, Huosheng $\mathrm{Hu}$

PII: S1566-2535(17)30270-1

DOI: 10.1016/j.inffus.2017.04.006

Reference: INFFUS 868

To appear in:

Information Fusion

Received date:

2 December 2016

Revised date:

31 March 2017

Accepted date:

22 April 2017

Please cite this article as: Sen Qiu, Zhelong Wang, Hongyu Zhao, Kairong Qin, Zhenglin Li, Huosheng Hu, Inertial/Magnetic Sensors based Pedestrian Dead Reckoning by Means of Multi-sensor Fusion, Information Fusion (2017), doi: 10.1016/j.inffus.2017.04.006

This is a PDF file of an unedited manuscript that has been accepted for publication. As a service to our customers we are providing this early version of the manuscript. The manuscript will undergo copyediting, typesetting, and review of the resulting proof before it is published in its final form. Please note that during the production process errors may be discovered which could affect the content, and all legal disclaimers that apply to the journal pertain. 


\section{Highlights}

- EKF approach is applied to reduce uncertainty and produce better estimations for PDR.

- A clustering algorithm is proposed to accurately distinguish stance phases.

- Sensor installation errors and path integral errors are properly corrected.

- The multi-sensor fusion method has been proved effective by optical apparatus. 


\title{
Inertial/Magnetic Sensors based Pedestrian Dead Reckoning by Means of Multi-sensor Fusion
}

\author{
Sen Qiu ${ }^{\mathrm{a}, \mathrm{b}}$, Zhelong Wang ${ }^{\mathrm{a}, *}$, Hongyu Zhao ${ }^{\mathrm{a}}$, Kairong Qin ${ }^{\mathrm{b}}$, Zhenglin Li ${ }^{\mathrm{c}}$, Huosheng \\ $\mathrm{Hu}^{\mathrm{d}}$ \\ ${ }^{a}$ School of Control Science and Engineering, Dalian University of Technology, Dalian, 116024, China \\ ${ }^{b}$ Department of Biomedical Engineering, Dalian University of Technology, Dalian, 116024, China \\ ${ }^{c}$ Dalian Municipal Central Hospital Affiliated Of Dalian Medical University, Dalian, 116033, China \\ ${ }^{d}$ Faculty of Computer Science and Electronic Engineering, University of Essex, Colchester, CO4 3SQ, UK.
}

\section{Abstract}

The challenges of self-contained sensor based pedestrian dead reckoning (PDR) are mainly sensor installation errors and path integral errors caused by sensor variance, and both may dramatically decrease the accuracy of PDR. To address these challenges, this paper presents a multi-sensor fusion based method in which subjects perform specified walking trials at self-administered speeds in both indoor and outdoor scenarios. After an initial calibration with the reduced installation error, quaternion notation is used to represent three-dimensional orientation and an extend Kalman filter (EKF) is deployed to fuse different types of data. A clustering algorithm is proposed to accurately distinguish stance phases, during which integral error can be minimized using Zero Velocity Updates (ZVU) method. The performance of proposed PDR method is evaluated and validated by an optical motion tracking system on healthy subjects. The position estimation accuracy, stride length and foot angle estimation error are studied. Experimental results demonstrate that the proposed self-contained inertial/magnetic sensor based method is capable of providing consistent beacon-free PDR in different scenarios, achieving less than $1 \%$ distance error and end-to-end position error.

Keywords: Body sensor network, multi-sensor fusion, pedestrian dead-reckoning, inertial/magnetic sensors

${ }^{*}$ Corresponding author: Zhelong Wang. Sen Qiu and Zhelong Wang contributed equally to this work and are co-first authors.

Email address: wangzledlut. edu.cn (Zhelong Wang) 


\section{INTRODUCTION}

PDR by using wearable inertial/magnetic sensors has been a research hotspot in safety application, medical monitoring, intelligent space and other commercial applications. PDR implies continuous location of pedestrian by quantification of human

5 lower limbs locomotion. Extensive researches have been conducted on how to fuse inertial/magnetic sensor measurements for accurate orientation and position estimation. Providing accurate estimation of pedestrian orientation and location information for both indoor and outdoor scenes is of interest to a wide variety of application domains, including:

- Emergency and Safety: PDR systems provide emergency services with real-time orientation and position information of rescue teams in areas with low visibility such as smoke-filled buildings or underground stations.

- Military and defense: Localization and information technologies are used by soldiers to self-locate, collect, collate, and convey information.

- Intelligent spaces and resource-efficiency: In order to fulfill the goal of intelligent spaces, it is essential to gain continuous location of individuals indoors.

Use cases even extend into motion monitoring in patients rehabilitation with chronic lower limbs diseases, and performance evaluation in the fields of competitive sport and digital entertainment industries.

\subsection{Related Work}

Various types of techniques have been introduced to implement PDR. PDR systems using pedometer existed but they can only be used to measure linear distance traveled. A prevalent practice is to deploy Global Positioning System (GPS), which is limited by low precision $(5 \sim 30$ meters) and can not meet the requirement of pedestrians who 25 might walk in sidewalks, public squares and even tunnels[1, 2, 3]. Especially, GPS signals are unavailable in indoor scenes where most individuals spend the majority of their time. However, the positioning of a subject on the correct floor in a multistory 
building can be achieved on the premise of accurate position and orientation estimation [4]. To address these limitations, PDR has been investigated using ultrasound or vision technologies in highly specialized laboratory containing expensive equipment, which was complicated and requires specially trained operators. The time and cost required to setup, deploy and maintain these equipment have prohibited routine deployment consequently. Adopting semantic properties or tags into the building fabric to act as landmarks is another solution, Xiao et al. [5] provides a framework for context-aware navigation services for vision impaired people, integrating intelligence into navigation environment; Millonig et al. [6] rely on landmarks to identify pedestrian flows and to imply landmark information into navigation services for pedestrians. These methods merely work well in modified buildings and cannot be rapidly deployed in arbitrary locations, i.e. in many cases beacon-free solutions are preferable since they do not depend on a pre-installed infrastructure. Other researchers have deployed customized communications systems such as GSM, WiFi, and RFID [7], which tend to result in sub-optimal localization on account of the limited communications access points and coverage overlap, and accuracies of several meters are normally reported.

In short, the aforementioned platforms and systems tend to be impractical for routine applications. The burgeoning development of wearable technology has offered a new solution to meet the demands of accurate and beacon-free PDR. Innovation of compact and low cost inertial/magnetic sensor module offer an alternative to existing PDR methods. Wearable inertial/magnetic sensors are portable and easy to maintain, allowing PDR to be carried out ambulatory and eliminating site-specific constraints. Meanwhile, this kind of human-centered application can be boosted by combining the emerging body sensor network (BSN) $[8,9]$ and sensor fusion technology [10]. As an interdisciplinary technology, wearable sensor network composed of micro-electromechanical systems, power electronics, control theory and computer technology is a perfect blend of fashion, convenience and interconnection [11]. Specifically, the embedded triaxial accelerometer can be employed as an superior pedometer and the magnetometer as a heading information source. Both [12] and [13] adopted smart-phones(with embedded inertial/magnetic sensors ) combined with floor plans for indoor location tracking, which are convenient for fast operation but the location 
accuracy is limited, besides, there will always be uncertain heading offsets between the smartphone orientation and the user's moving direction.

In literature, many self-contained methodologies have been proposed for PDR based on wearable sensors. These methodologies integrate step lengths and orientation estimations at each detected step, so as to compute the absolute position and orientation of a pedestrian. Godha and Lachapelle [14] proposed a foot mounted inertial system to evaluate pedestrian dead reckoning system performance under challenging GPS conditions. Some PDR approaches assume a smooth walk on horizontal surfaces [15], and others are valid for uneven terrain, Park et al. [16] even conducted PDR on running trials and detected zero velocity intervals accurately.

Hybrid systems are gaining more attention recently. Widyawan et al. [17] proposed multimoding sensor data fusion for robust navigation in unknown environments. Faragher et al. [18] developed a hybrid indoor navigation system that is independent of a centrally-established database and pre-supplied building map. Hybrid systems seem to be the most promising solution but they introduce additional complexity to the system and multidisciplinary fusion is still a issue with great challenge [19].

An ideal PDR would be conducted on condition that both walking velocity and heading angle are accurately determined at all instants for positions to be determined. However, both previous values and variable quantities are estimated rather than determined accurately, which inevitably causes large errors. PDR positioning accuracy normally ranges from $0.3 \%$ to $10 \%$ of the total travelled distance [20], but the accuracy of implemented algorithm strongly depends upon the road condition, travel time, sensor performance, magnetic disturbances and several other factors. The aim of this paper is to develop a method which merely relies on self-contained inertial/magnetic sensor for accurate estimation of PDR, as well as validate an improved ZVU method for PDR against an off-the-shelf optical motion tracking system.

\subsection{Outline}

In this paper, we intend to adopt shoe-mounted inertial/magnetic sensors to achieve self-contained PDR with minimized error in different scenarios without aiding from any other complementary technologies. An effective approach was proposed to tackle 
the issue of pedestrian navigational errors due to sensor misplacement and sensor variance, which are only driven by data from the inertial/magnetic sensor. With the aim of applying the algorithm into different scenarios with limited on-board computational ability, an wearable sensor based pedestrian dead reckoning method is adopted. In our framework, the inertial/magnetic sensor is regarded as the proprioceptive sensor, which provides the state prediction under EKF; the available ground truth from optical system serves as a tool for algorithm verification.

This paper is structured as follows: problem formulation and multi-sensor fusion methodology are first introduced in section II, followed by the experimental analysis in section III; the discussion and a brief comparison with recent work will be presented in section IV; while section V gives the conclusion.

\section{PROBLEM FORMULATION AND MULTI-SENSOR FUSION METHOD- OLOGY}

In general, pedestrian dead reckoning systems track three-dimensional position and orientation by estimating the complete trajectory of the attached sensor system [21]. Orientation is normally updated by sensing gravity and geomagnetic field. Meanwhile, the updates of position are based on path integration, which is the process of calculating current position of a pedestrian by using a previously determined position and advancing that position based on the speed over elapsed time. Similarly, animals such as ants and rodents are able to estimate their current location relative to their nest continuously based on their movements from their last known location. As for PDR, walking speed can be obtained by integrating the corresponding linear acceleration which can be quantified by accelerometer on condition that the effects of gravity are properly deducted. The numerical integration process is prone to cumulative errors in that each position estimation is relative to the previous process. The widely adopted ZVU method still rely on the accurate definition of stance phase interval, which remains a challenge in this 115 field. Moreover, issues still exist regarding false stance phase detection, and optimal sensor positioning.

In consideration of the aforementioned problems, the performance of PDR can be 


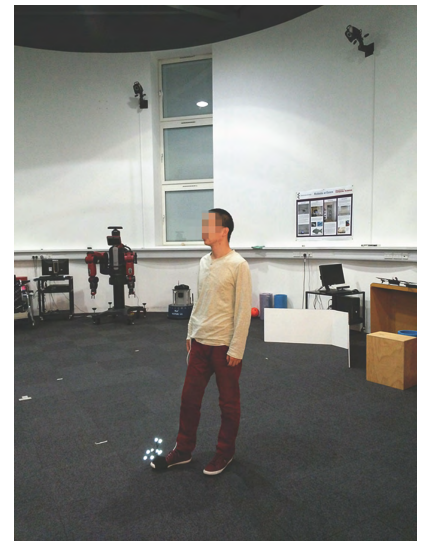

(a) Experiment in Essex Robot Arena

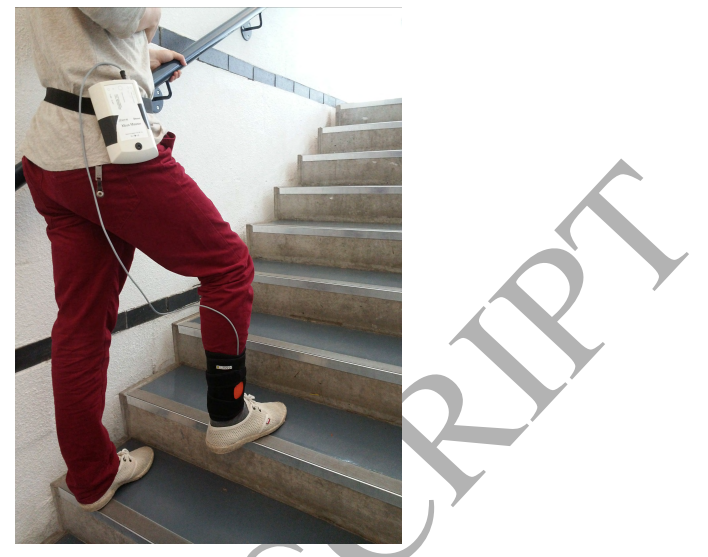

(b) Experiment in the stairwell

Figure 1. Sensors mounted on the subject at different location in Robot Arena and stairwell

improved significantly with a definite initial state and proper corrections through the journey. In this study, PDR is conducted by an ambulatory wearable sensor system.

The measurements are provided by an inertial/magnetic sensor system made by Xsens attached to the foot, as shown in Fig. 1. No pre-installed infrastructure is needed for this system, while an optical motion capture system named Vicon was adopted in this study just for validation of proposed method. Hence, each data set is composed of two data subsets: one from body attached sensors including three-dimensional (3D) linear acceleration, angular speed and magnetic field intensity, and the other data subset from Vicon system including 3D position and 3D orientation, i.e. the ground truth. Besides, Xsens system directly output 3D orientation but the output is prone to error caused by magnetic disturbance.

The performance specification of Xsens sensor system is presented in Table 1. Each inertial/magnetic sensor consists of a triaxial accelerometer, a triaxial gyroscope and a triaxial magnetometer. Raw sensor data is logged to the data logger placed on the waist at a sampling rate of $100 \mathrm{~Hz}$, and the data can be forwarded to a receiver via Bluetooth. The wearable sensors were fitted with stretchable hook-and-loop fastener for steady fastening and user comfort during the experiment. 
Table 1. SENSOR PERFORMANCE SPECIFICATION

\begin{tabular}{cccc}
\hline \hline Unit & Accelerometer & Gyroscope & Magnetometer \\
\hline Dimensions & 3 axes & 3 axes & 3 axes \\
Dynamic Range & $\pm 50 \mathrm{~m} / \mathrm{s}^{2}$ & $\pm 1200 \mathrm{deg} / \mathrm{s}$ & $\pm 750 \mathrm{mGauss}$ \\
Bandwidth (Hz) & 30 & 40 & 10 \\
Linearity (\% of FS) & 0.2 & 0.1 & 0.2 \\
Bias stability (unit 1 $\sigma)$ & 0.02 & 1 & 0.1 \\
Alignment Error (deg) & 0.1 & 0.1 & 0.1 \\
\hline \hline
\end{tabular}

Problems arise due to the variability in sensor positioning for the same trial across different subjects and for the same individual. There are often cases when the sensor frame are not axis aligned to the subject whose orientation needs to be estimated, however, the target is to record the sensor data in the body coordinate rather than the sensor coordinate. To tackle this problem, after the sensor installation, we calculated the initial orientation and the initial rotation matrix $R_{\text {trans }}$ which performs the initial transformation of the sensor frame. In this case, the sensor data are expressed in the body coordinate as targeted.

Initial orientations are usually determined by standard gravity and local magnetic field. Specifically, initial pitch and roll can be calculated by sensing the gravity vector at rest using accelerometers. Due to the inability of an accelerometer to sense the heading angle change in the $\mathrm{X}-\mathrm{O}-\mathrm{Y}$ plane. A magnetometer provides the other reference vector $\left[m_{x}, m_{y}, m_{z}\right]^{T}$. Other than gravity which is vertically downward, there is an angle between earth magnetism vector and the X-O-Y plane. i.e. the magnetic inclination indicated by $\beta$ in Fig. 2(a). On condition that the sensor orientation of the sensor is adjust to north, the vector turn to $\left[m_{x}, 0, m_{z}\right]^{T}$. In this paper, gravity vector is first used to rotate the body frame to the same $\mathrm{X}-\mathrm{O}-\mathrm{Y}$ plane of ground frame then the magnetometer is adopted to calculate the difference of heading. 


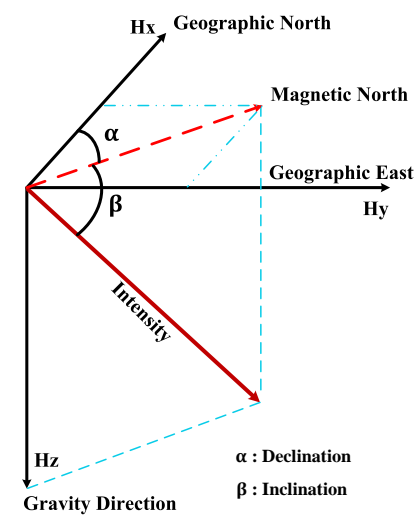

(a) Magnetic declination and inclination

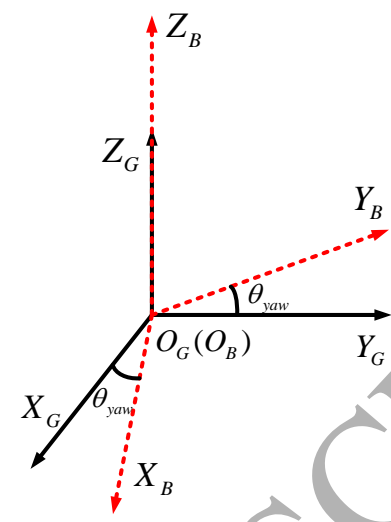

(b) Heading estimation by magnetometer

Figure 2. Geomagnetic component and the heading determination

The initial roll angle $\phi$, pitch angle $\theta$ and heading angle $\psi$ can be calculated in formula (1).

$$
\left\{\begin{array}{l}
\phi=\arctan \left(a_{y}, a_{z}\right) \\
\theta=\arctan \left(-a_{x}, a_{y} * s \phi+a_{z} * c \phi\right) \\
\psi=\arctan \left(m_{y} * c \phi+m_{x} * s \phi * s \theta\right. \\
\left.-m_{z} * s \phi * c \theta, m_{x} * c \theta-m_{z} * s \theta\right)
\end{array}\right.
$$

where $c$ and $s$ denote cosine and sine functions respectively. $a_{x}, a_{y}$ and $a_{z}$ represent 155 acceleration magnitudes, $m_{x}, m_{y}$ and $m_{z}$ are magnetometer measurements.

The next issue is to calculate the initial rotation matrix $N_{\text {ini }}$ which performs the

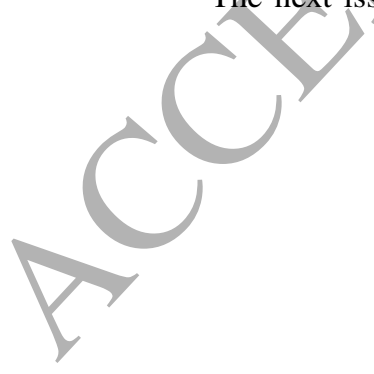




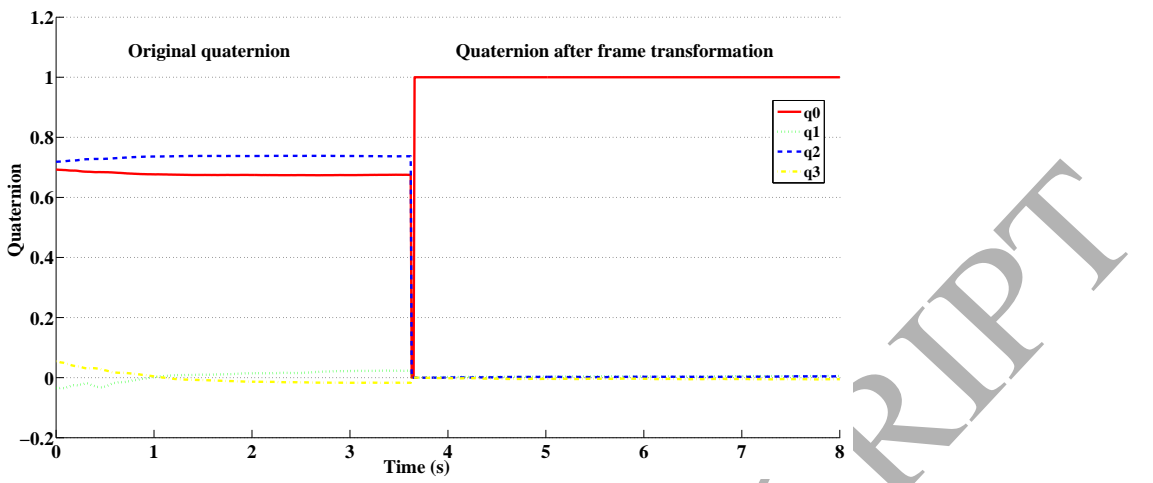

Figure 3. Quaternion update using proposed initial alignment method

sensor frame transformation.

$$
\begin{aligned}
N_{i n i} & =R_{\psi} R_{\theta} R_{\phi} \\
& =\left[\begin{array}{ccc}
c \psi & -s \psi & 0 \\
s \psi & c \psi & 0 \\
0 & 0 & 1
\end{array}\right]\left[\begin{array}{ccc}
c \theta & 0 & s \theta \\
0 & 1 & 0 \\
-s \theta & 0 & c \theta
\end{array}\right]\left[\begin{array}{ccc}
1 & 0 & 0 \\
0 & c \phi & -s \phi \\
0 & s \phi & c \phi
\end{array}\right] \\
& =\left[\begin{array}{ccc}
c \theta c \psi & s \phi s \theta c \psi-c \phi s \psi & c \phi s \theta c \psi+s \phi s \psi \\
c \theta s \psi & s \phi s \theta s \psi+c \phi c \psi & c \phi s \theta s \psi-s \phi c \psi \\
-s \theta & s \phi c \theta & c \phi c \theta
\end{array}\right]
\end{aligned}
$$

Fig. 3 shows an example of initial alignment process, the initial quaternion $q_{0}$ becomes close to $(1,0,0,0)$ when the subject was at rest by conducting the alignment operation. While Table 2 shows the elements of the quaternion for the six key samples. The ideal orientation of the sensor after initial alignment is represented by the quaternion $(1,0,0,0)$ in the navigation frame.

By applying the proposed initial alignment, the sensor data would be expressed in the body coordinate whose orientation is supposed to be recorded. The new $X$ axis in ground frame is chosen by the advancing direction of the subject while keeping the $Z$ axis straight up. Y axis can be obtained according to right handed Cartesian coordinate 165 system. 
Table 2. QUATERNION UPDATES WITH INITIAL ALIGNMENT

\begin{tabular}{ccccc}
\hline \hline Sample & $q_{0}$ & $q_{1}$ & $q_{2}$ & $q_{3}$ \\
\hline 1 & 0.67538 & 0.02347 & 0.73714 & -0.01705 \\
2 & 0.67525 & 0.02364 & 0.73746 & -0.01784 \\
3 & 0.67549 & 0.02351 & 0.73723 & -0.01738 \\
4 & 0.99998 & -0.00032 & 0.00459 & -0.00132 \\
5 & 0.99998 & -0.00072 & 0.00370 & -0.00139 \\
6 & 0.99999 & -0.00019 & 0.00416 & -0.00155 \\
\hline \hline
\end{tabular}

\subsection{Multi-sensor fusion based orientation updates}

As a nonsingular description of 3D orientation, quaternion is more efficient than direction-cosine matrix and Euler angles methods, and quaternion is unique up to sign. A unit quaternion vector with unit magnitude can be interpreted to indicates a rotation about a unit vector $\vec{n}$ through angle $\theta$

$$
\vec{q}=\cos \frac{\theta}{2}+\sin \frac{\theta}{2} \cdot \vec{n}
$$

An alternative representation of a quaternion is a vector with a real component $q_{0}$ and a complex part, which can be described in the following vector format:

$$
\vec{q}=q_{0}+q_{1} \vec{i}+q_{2} \vec{j}+q_{3} \vec{k}
$$

In this paper, the corresponding continuous nonlinear system can be expressed as follows:

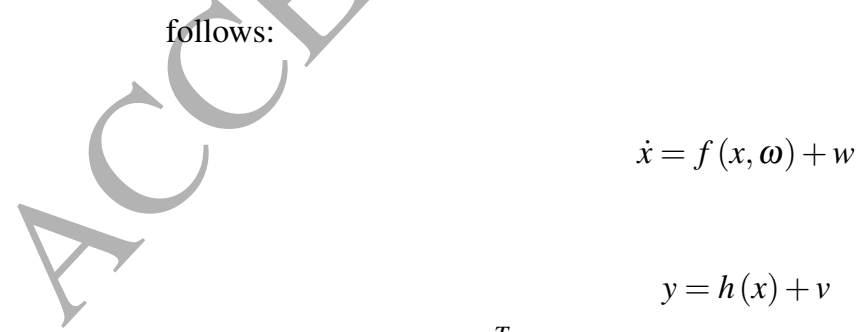

where $x=\left[\begin{array}{ll}\vec{q} & \vec{b}_{\omega}\end{array}\right]^{T}$ is the systematic state vector, and gyroscope bias is represented by $\vec{b}=\left[\begin{array}{lll}b_{\omega x} & b_{\omega y} & b_{\omega z}\end{array}\right]^{T}$, in which $\omega$ represents angular velocity vector 
$\left[\begin{array}{lll}\omega_{x} & \omega_{y} & \omega_{z}\end{array}\right]$, meanwhile, $y=[\vec{a}]=\left[\begin{array}{lll}a_{x} & a_{y} & a_{z}\end{array}\right]$ is the systematic observation vector. In this way, the rotary motion of rigid body can be discribed as

$$
\dot{q}=\left[\begin{array}{cc}
{[\vec{\omega} \times]} & \vec{\omega} \\
-\vec{\omega}^{T} & 0
\end{array}\right] \cdot q
$$

where

$$
[\vec{\omega} \times]=\left[\begin{array}{ccc}
0 & -\omega_{z} & \omega_{y} \\
\omega_{z} & 0 & -\omega_{x} \\
-\omega_{y} & \omega_{x} & 0
\end{array}\right]
$$

with $[\vec{\omega} \times]$ representing the skew-symmetric matrix. The inertial/magnetic sensor variances are assumed as random walk process, which is driven by zero-mean Gaussian models, denoted as $\vec{v}_{a}, \vec{v}_{g}$ and $\vec{v}_{m}$, respectively. The real angular velocity, real acceleration and the magnetic field are related with gyroscope, accelerometer and magnetometer measurements in the following quaternion kinematic matrix.

$$
\left\{\begin{array}{l}
\vec{\omega}=\omega_{r}+\vec{b}_{\omega}+\vec{v}_{g} \\
\vec{a}=q_{N}^{S}\left(a_{a} \overrightarrow{+} g\right) q_{N}^{S-}+\vec{v}_{a} \\
\vec{h}=q_{N}^{S} h_{m} q_{N}^{S-}+\vec{v}_{m}
\end{array}\right.
$$

where $\vec{v}_{a}, \vec{v}_{g}$ and $\vec{v}_{m}$ are noise signals of accelerometer, gyroscope and magnetometer, respectively. As defined here $q_{N}^{S}$ rotates a vector in the navigation frame to the sensor frame, and $q_{N}^{S-}$ is the corresponding complex conjugate.

$$
\vec{g}=\left[\begin{array}{lll}
0 & 0 & g
\end{array}\right]^{T}
$$

Nonlinear equations $f(x, \omega)$ and $h(x)$ can be expressed as follows;

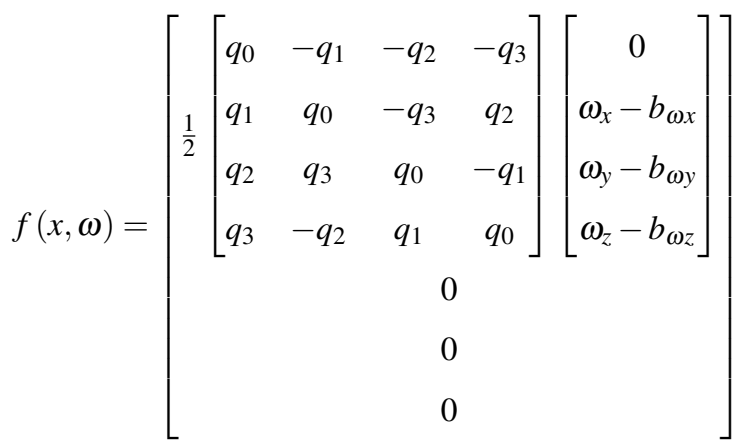




$$
h(x)=\left[\begin{array}{ll}
C_{N}^{S}(q) & 0_{3 \times 3}
\end{array}\right]\left[\begin{array}{c}
\vec{g} \\
0_{3 \times 1}
\end{array}\right]
$$

where the partial derivative of $f(x, \omega)$ with respect to vector $x$ can be written as

$$
A=\frac{1}{2}\left[\begin{array}{ccccccc}
0 & -\omega_{x}+b_{\omega x} & -\omega_{y}+b_{\omega y} & -\omega_{z}+b_{\omega z} & q_{1} & q_{2} & q_{3} \\
\omega_{x}-b_{\omega x} & 0 & \omega_{z}-b_{\omega z} & -\omega_{y}+b_{\omega y} & -q_{0} & q_{3} & -q_{2} \\
\omega_{y}-b_{\omega y} & -\omega_{z}+b_{\omega z} & 0 & \omega_{x}-b_{\omega x} & -q_{3} & -q_{0} & q_{1} \\
\omega_{z}-b_{\omega z} & \omega_{y}-b_{\omega y} & -\omega_{x}+b_{\omega x} & 0 & q_{2} & -q_{1} & -q_{0} \\
0 & 0 & 0 & 0 & 0 & 0 & 0 \\
0 & 0 & 0 & 0 & 0 & 0 & 0 \\
0 & 0 & 0 & 0 & 0 & 0 & 0
\end{array}\right] \text { (13) }
$$

where the partial derivative of $h(x)$ with respect to vector $x$ can be written as

$$
H=g \cdot\left[\begin{array}{ccccccc}
-2 q_{2} & 2 q_{3} & -2 q_{0} & 2 q_{1} & 0 & 0 & 0 \\
2 q_{1} & 2 q_{0} & 2 q_{3} & 2 q_{2} & 0 & 0 & 0 \\
2 q_{0} & -2 q_{1} & -2 q_{2} & 2 q_{3} & 0 & 0 & 0
\end{array}\right]
$$

The error covariance matrix can be written as

$$
\hat{P}_{k}=A_{k-1} P_{k-1} A_{k-1}^{T}+Q
$$

When observed values are available, i.e., stance phase is detected, the accelerometer measurement (acceleration of gravity) can be used to correct the estimated systematic state. The correction of $\vec{a}$ can be written as

$$
\begin{gathered}
x_{k}=\hat{x}_{k}+K_{k}\left(\vec{a}_{k}-H \vec{g}\right) \\
P_{k}=\left(I_{7 \times 7}-K_{k} H_{k}\right) \hat{P}_{k}
\end{gathered}
$$

where $K_{k}$ is the kalman filter gain, which can be calculated by

$$
K_{k}=\hat{P}_{k} H_{k}^{T}\left(H_{k} \hat{P}_{k} H^{T}+R\right)^{-1}
$$

The EKF filter described in this section continuously build the sensor variance model and makes the corrections. In this way, path integral errors caused by sensor variance could be minimized, which is presented in the following sections. 


\subsection{Stance Phase detection}

Each step we take when we walk can be segmented into several phases which form the gait cycle. The primary phases are stance and swing. Provided that at least one foot is in contact with the ground at any given moment during normal walking, i.e., no flight phase exists when running. The foot to which the sensor is attached stands steady on the ground in the stance phase. In the swing phase, the corresponding foot uplifts from behind the pedestrian, swings forward and ends at the next stance phase. The foot then uplifts from behind the pedestrian before swinging forward again for the next stance phase. As shown in Fig. 4. According to literature, the foot is in stance phase for roughly $60 \%$ of the walking time and swing phase take up for the remaining $40 \%[14][22]$.

In general, the fundamental cycle of a pedestrian dead reckoning systems includes:

- Identify data segmentations of the walking motion information corresponding to individual steps.

This is achieved by detecting cycles in the sensor data caused by the repetitive motion of walking. This involve searching for repeating data patterns, the inertial measurement peak values due to heel-strike are chosen as judgment index in this paper. Experimental results show that they are well suited to step segmentation.

- Determine the stance phase and swing phase in each gait cycle.

Three indicators were evaluated to determine stance phase as detailed in this section. Meanwhile, a simple K-mean clustering algorithm is proposed to distinguish true stance phases from false stance phases.

- Estimate the foot velocity, foot position and heading angle using sensor data step by step, which is the process of estimating the value of any variable quantity by using an earlier value and adding whatever changes have occurred in the meantime.

Gyroscope has been deemed to be more dependable than accelerometer in algorithmical stance phase detection [23]. In this paper, three indicators are deployed to determine stance phase when the subject stands steady on the ground (usually lasts 


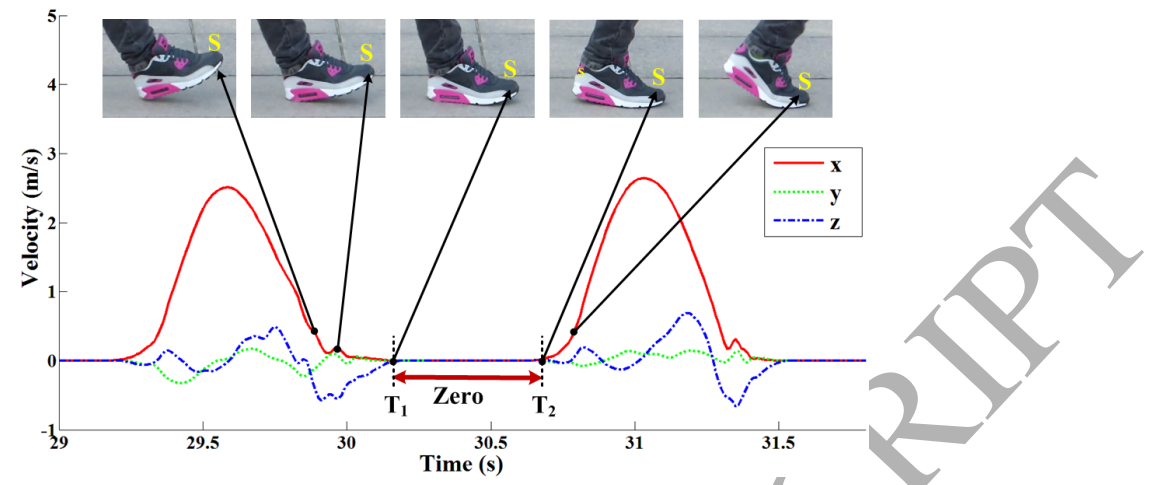

Figure 4. A typical stance phase between two consecutive swing phase

$0.2 \sim 0.4$ seconds in each step). Stationary states are calculated by taking the squared Euclidean norm of acceleration in formula (19),

$$
A=\sqrt{\left(a_{x} /\|\mathbf{g}\|\right)^{2}+\left(a_{y} /\|\mathbf{g}\|\right)^{2}+\left(a_{z} /\|\mathbf{g}\|\right)^{2}}
$$

where $a_{x}, a_{y}$ and $a_{z}$ represent the triaxial acceleration measurements.

It is assumed that the measurements of triaxial accelerometer are essentially constant in stance phase and the magnitude of composed acceleration vector is approximately equal to local grayity, in this case, the acceleration moving variance $\mathrm{M}$ is deployed as the second indicator (20),

$$
M=\frac{1}{N} \sum_{i=j-N}^{i=j}\left(\left(S_{i}-\bar{S}_{N}\right)^{2}\right.
$$

where $\bar{S}_{N}$ is the mean of $S_{i}$ over $N$ samples,

Meanwhile, angular rate energy $E_{\text {gyro }}$ is adopted as the third indicator (21). The second origin moment rather than second central moment is used to detect stance phase.

As defined in the following energy detector[23]:

$$
\begin{gathered}
E=\frac{1}{\sigma_{\omega}^{2} W} \sum_{i=j}^{j+W-1}\left\|\omega_{i}\right\|^{2} \\
\widehat{R}=\left\{\begin{array}{c}
1, A<\lambda_{1} \cap M<\lambda_{2} \cap E<\lambda_{3} \\
0, \text { other value }
\end{array}\right.
\end{gathered}
$$


where $W$ is the window size selected according to the sensors sampling rate; $\omega_{i}=$ $\left[\omega_{x, i}, \omega_{y, i}, \omega_{z, i}\right]^{T}$ is the triaxial angular velocity vector, $\sigma_{\omega}^{2}$ is the gyroscope noise variance. $\lambda_{1}, \lambda_{2}$ and $\lambda_{3}$ are empirically predefined thresholds. The detection results $(\widehat{R})$ are sequences consist of "zero" and "one". The algorithm continually finds the interval when ZVU is valid and updates the corresponding $\mathbf{v}_{G}(t)$ as $[0,0,0]^{T}$ base on the above three indicators.

In consideration of potential short-term sensor measurement fluctuations, time constraint is a good supplement to verify the stance phase detection results by evaluating the time durations of each phase. The principle is that a true stance phase can be declared only when the detection statistics fall in specified period of time. In general, a fixed time constraint only works well for the specific pedestrian data, which is not suitable for pedestrian data of different walking scenarios. Therefore, an adaptive time constraint is required to provide robust PDR solutions. In this paper, the k-mean clustering algorithm is adopted to filter potential gait phases and automatically yield the adaptive time constraint parameters. As illustrated in Fig. 5, the k-mean clustering algorithm efficiently classifies the detected stance phases into true and false clusters according to time durations, so that the fluctuation-induced false stance phases could be eliminated. Meanwhile, the extreme values of each cluster are illustrated by blue line shown in Fig. 5. In this case, the k-mean algorithm can yield adaptive time constraint parameters to eliminate the false stance phase detection due to sensor measurement fluctuations. Fig. 6 shows the effect of the window size $W$ on the step number detection. 25 different window size are selected, i.e., from 1 to 25 , and the correct step number is 56 in the trial. It turned out that the optimal window size is neither too big nor too small.

\subsection{Zero-velocity-update-aided pedestrian dead reckoning}

In this paper, the basic linear physics of walking movement is as follows:

$a_{k} \in R^{3 \times 1}:$ acceleration in sensor coordinate

$v_{k} \in R^{3 \times 1}$ : velocity in the ground navigation coordinate

$p_{k} \in R^{3 \times 1}:$ position in the ground navigation coordinate

235

$\omega_{k} \in R^{3 \times 1}$ : angular velocity in sensor coordinate 


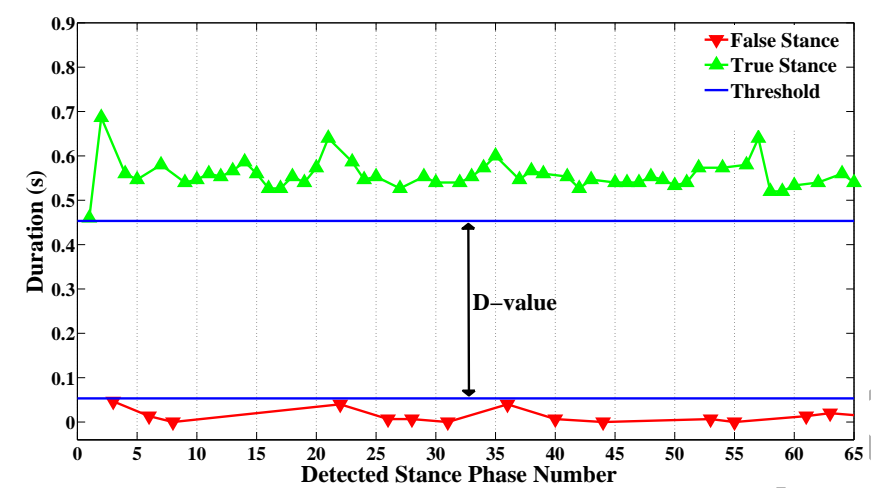

Figure 5. Using K-mean cluster to clasify ture and false stance phases

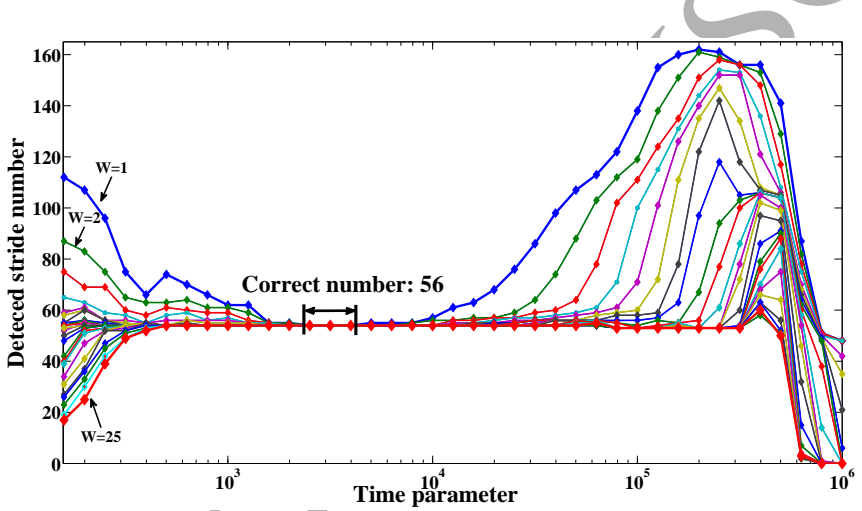

Figure 6, Using different window size to detect step number

And the kinematics equations of PDR for a discrete system are as follows:

$$
\left[\begin{array}{c}
a_{k} \\
v_{k} \\
p_{k}
\end{array}\right]=\left[\begin{array}{c}
q_{k-1} a_{k-1} q_{k-1}^{*} \\
v_{k-1}+\frac{\tau}{2}\left(a_{k}+a_{k-1}-2 \mathbf{g}\right) \\
p_{k-1}+\frac{1}{2}\left(v_{k-1}+v_{k}\right) \tau+\frac{1}{2} a_{k-1}(\tau)^{2}
\end{array}\right]
$$

where $k$ represents the time index, $\tau$ denotes the sampling interval (10 milliseconds in this paper), $\mathbf{g}$ is the local gravity, Further, $q_{k}$ is the quaternion describing the orientation of the system, the triple product $q_{k-1} a_{k} q_{k-1}^{*}$ denotes the rotation of $a_{k}$ by ${ }_{240} q_{k}$.

This formula is used to move the foot until a new update is received from the iner- 
tial/magnetic sensors. The sensor measurement noise is inevitable and constantly accumulated because position information is estimated in a cumulative way, which leads to deviation from the ground truth. Generally, foot velocity is obtained by numerically integrating corrected foot acceleration measurements obtained during the swing phase. Accurate foot velocity estimation is the premise of position estimation. To achieve accurate pedestrian dead reckoning of an subject equipped with foot-mounted sensors, widely used ZVU method was adopted to correct the integral error and inherent sensor variance error at the end of each step. ZVU was first introduced in a PDR context in the NavShoe project by Foxlin [24]. It is convenient to incorporated ZVU into the PDR system by formulating it as pseudomeasurements of zero velocity. ZVU must only be implemented when the foot is completely at the stance phase[25][26].

In this case, integration of inertial data only occur in the swing phase, reducing the integral error of position estimation of the foot to which the sensor is attached. Note that the accelerometer measures all forces that are applying on the sensor including gravity, which is irrelevant to foot locomotion. In this case gravity vector should be deducted properly. Assuming that the bias error of accelerometer in a gait cycle is stable, we set the foot velocity to zero at the end of each swing phase based on the periodicity of walking movement.

The foot acceleration in dynamic phase can be expressed as

$$
a_{N}(t)=\tilde{a}_{N}(t)+\varepsilon
$$

where $\tilde{a}_{N}$ is the true motion acceleration, $\varepsilon$ is the bias error of the accelerometer. Assuming that the bias error is fixed in one gait cycle. Then The foot velocity is supposed to be zero during the whole stance phase. And the dynamic phase speed can be calculated by the formula:

$$
\begin{aligned}
& v_{N}(t)=\int_{0}^{t} a_{N}(\tau) d \tau=\int_{0}^{t}\left[\tilde{a}_{N}(\tau)+\varepsilon\right] d \tau \\
& =\tilde{v}_{N}(t)+\varepsilon t
\end{aligned}
$$

The bias error of acceleration during a gait cycle can be estimated as

$$
\varepsilon=\frac{v_{N}(T)}{T}
$$



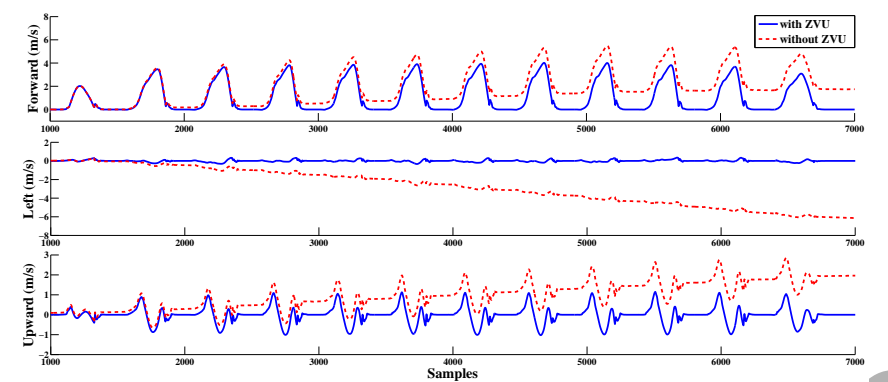

Figure 7. Foot velocity prior to applying ZVU (red dotted curve), and after applying ZVU (blue curve). The estimation of three-dimensional foot velocity: forward, left and upward (top to down)

where $T$ is the duration of dynamic phase.

After the foot velocity correction we can obtain corrected position information:

$$
p(t)=\int \tilde{v}_{N}(t) d t
$$

Fig. 7 shows the improvement of three-dimensional foot velocity estimation with ZVU. It is obvious that ZVU properly eliminates the integral error caused by sensor variance. Some researchers developed a smoother for velocity and position estimation[27], however, a delay would be introduced, which limits real time applications, and it is still controversial upon the real effect on PDR. The flowchart of the whole proposed approach is shown in Fig. 8 .

\section{EXPERIMENTAL/RESULTS AND ALGORITHM VALIDATION}

To assess the overall algorithm, we conducted experiments in two scenarios: indoor locomotion and outdoor positioning. For each scenario, we attached one Xsens inertial/magnetic sensor module on the subjects' instep. Four test subjects (two males and two females without a history of lower limb pathologic conditions) performed indoor rectangular level walking in robot arena and stair climbing trials in the stairwell, as well as outdoor trials performed in a 400 meters stadium track, with several repetitions of each walking trial. 


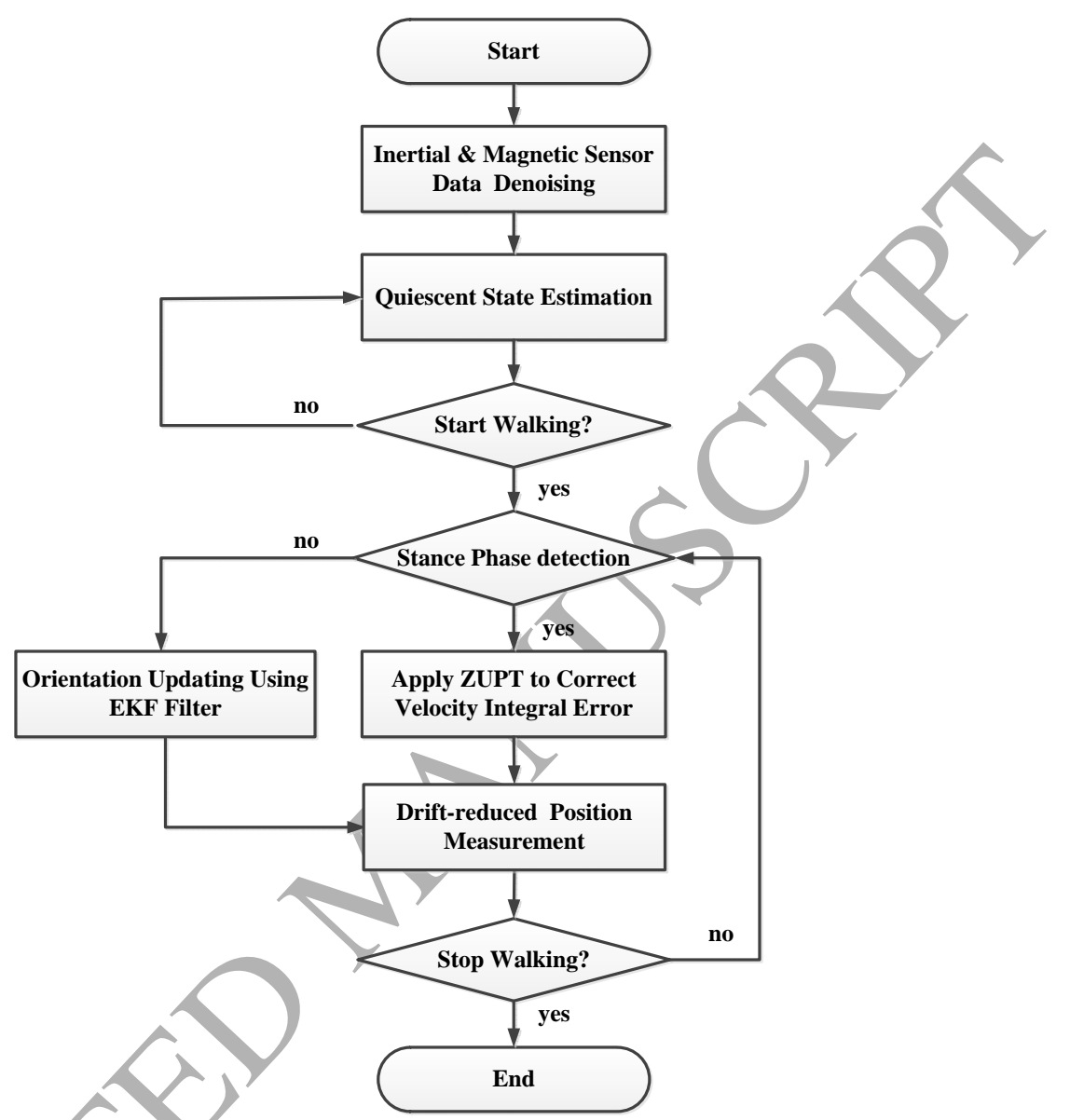

Figure 8. Flowchart of the proposed PDR method

\subsection{Indoor experimental results}

The subjects were instructed to walk along the predefined rectangular path in the robot arena, being covered by Vicon cameras, as shown in Fig. 9 The route is from the origin $(x=0, y=0, z=0)$ with zero heading angle, and then along the clockwise direction of the rectangular grid line for several laps, ending up at the origin, though 280 the route is not strictly required.

Fig. 10 (a) and Fig. 10 (b) outline 3D foot velocity of walking trial in Robot Arena and stairwell, respectively. No significant drift was observed from the two trials. Foot 


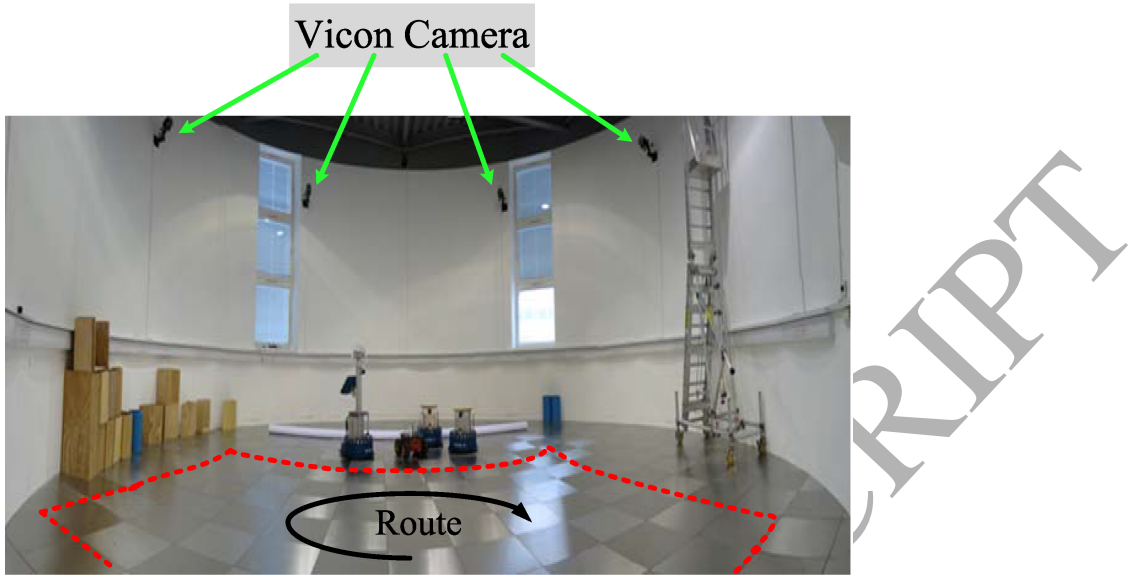

Figure 9. Indoor experimental scenario: the circular region in Essex robot arena

velocity in $\mathrm{Z}$ axis is the secondary components in level walking, when $\mathrm{X}$ axis and $\mathrm{Y}$ axis alternately become the main component. While $\mathrm{Z}$ axis foot velocity becomes the fundamental component in the whole process of stair climbing because the subject was keep rising up during the trial. Likewise, 3D foot position of two types of indoor experiment presents the same trend. The dominant foot that the senor was attached on started from the origin and then moved along the rectangular route clockwise for two laps, ending up at $(0.125,0.038,0.011)$ with a reverse heading angle (heading = $290-177.3^{\circ}$ ). Note that Vicon system does not work in staircase, so we took the layer height (0.16 meters) and width (0.3 meters) as ground truth instead. In Fig. 10 (c), the $3 \mathrm{D}$ foot position ends at $(0.022,-1.094,3.305)$, which is reasonable compared with the available ground truth.

Moreover, the obtained foot velocity and position information can be useful for stair ascent capacity evaluation. The trajectories of two types of trials are demonstrated in Fig. 11 (a). and Fig. 11 (b), respectively. Pedestrian walking distance errors have always been the primary concerns in literature [15] [28] [29], while the limitation of evaluating the overall distance errors is the canceling effect of the positive and negative errors occur in each stride. From this perspective, it is worth studying how the stride length error changes in the course of trial. In general, a step represents the period or 


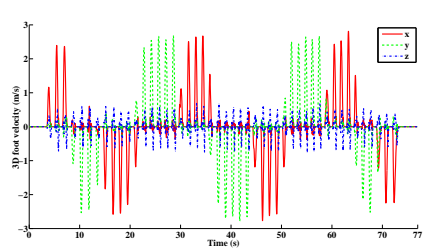

(a) 3D foot velocity of rectangular level walking in robot arena

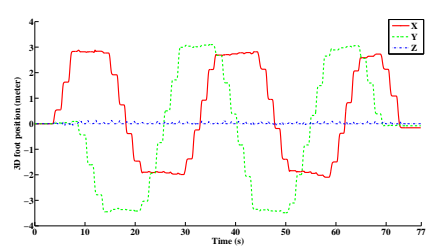

(c) 3D foot position of rectangular level walking in robot arena

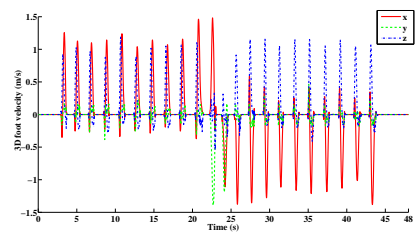

(b) 3D foot velocity of stair climbing

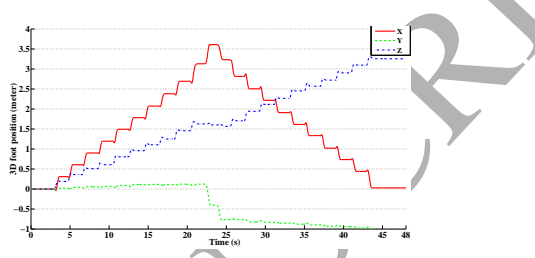

(d) 3D foot position of stair climbing

Figure 10. 3D foot velocity and position of walking trial in Robot Arena and stairwell

locomotion between two consecutive footfalls on opposite feet, while a stride is defined as the same quantity with respect to the same foot. In this paper, stride length is defined as the distance between the adjacent foot landing, which determine the walking speed by dividing the elapsed time in one stride. Towards level walking trials, stride length $\mathrm{S}$ can be calculated by

$$
S_{k}=\sqrt{\left(p_{x, k}-p_{x, k-1}\right)^{2}+\left(p_{y, k}-p_{y, k-1}\right)^{2}}
$$

where $S_{k}$ represents the stride length of $k$ th step. $p_{x, k}$ and $p_{y, k}$ denote the $X, Y$ coordinate component of foot position in navigation frame, respectively.

The stride length estimation results are analyzed. The overall estimation error in this trial is slightly larger $(0.41 \%)$ than that of Vicon, i.e., the ground truth. As for each stride, the calculated results are less than the true value for 7 steps and larger than the true value as to the remaining 21 steps. Maximum error appears in the 18th step with $0.0105 m(1.31 \%)$, and the $\mu$ and $\sigma$ are $0.482 \%$ and $0.376 \%$, respectively.

As can be seen from the bar chart, stride length errors are not likely to grow with the increase of step number based on the small sample experiments, though there are actually small increment when the subject changes walking direction, i.e., the moment 


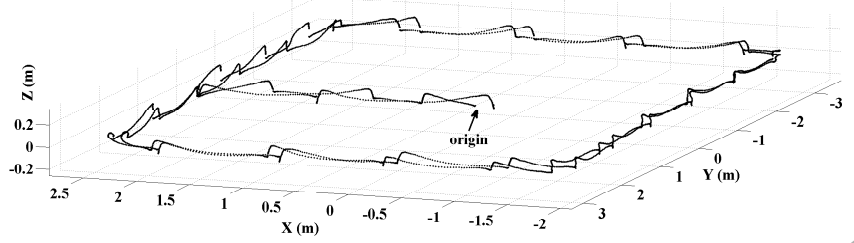

(a) Foot trajectory of rectangular level walking in robot arena

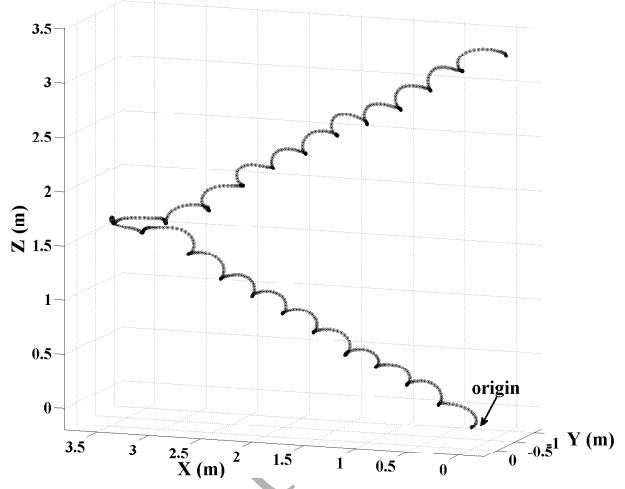

(b) Foot trajectory of stair climbing

Figure 11. Foot trajectory of walking trial in (a) Essex Robot Arena and (b) stairwell

during which the subject made the turning at each corner. Still we can draw that the

PDR method works well in level walking trials and the position estimation errors are well controlled.

To get a more clear understanding of error distribution, Fig. 12 indicates the corresponding frequency histogram, kernel density estimation and normal distribution fitting of stride length error by different method, including proposed EKF method, factored quaternion algorithm (FQA), Gradient descent algorithm (GDA) and complementary filter $(\mathrm{CF})$ method. The statistical magnitude of proposed EKF method are: mean value $\mu=0.0051$ and standard deviation $\sigma=0.0046$. While the results of comparative methods are: factored quaternion algorithm $(-0.016 \pm 0.022)$, Gradient descent algorithm 


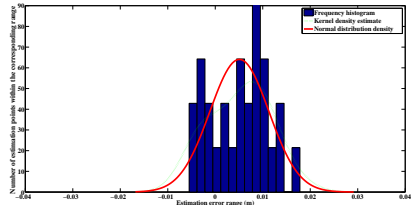

(a) Proposed EKF Method

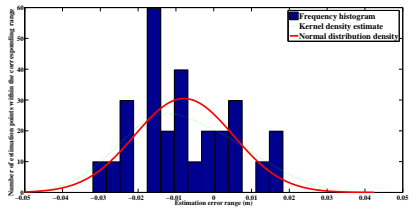

(c) Gradient descent algorithm[30]

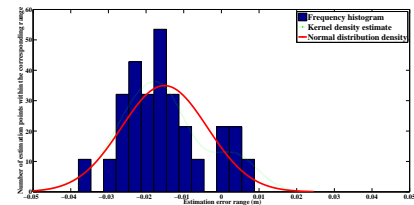

(b) Factored quaternion [28]

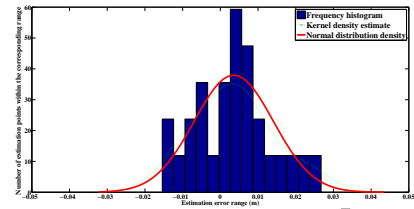

(d) Complementary filter method[29]

Figure 12. Stride estimation error distribution by different methods

$(-0.0086 \pm 0.017)$ and complementary filter algorithm $(0.0039 \pm 0.0083)$. Though the mean error of proposed method is not the smallest, the standard deviations are remarkable smaller than the rest methods, which reflects the effectiveness of error-controlling.

In order to investigate the repeatability of the proposed PDR method, we invited one subject to perform the rectangular walking trial for six times. The trial results are listed in Table 3. All steps in six trials are correctly detected with the proposed method for stance phase detection, and an average distance error of $0.41 \%$ for level walking was achieved within the indoor environment, as well as an average heading error of $0.52^{\circ}$ per turn. It is unreasonable to just compare the results against the literature because the system performance depends on several factors to a large extent, including system hardware, sensor placement, road conditions, etc. Even though, our experimental results are comparable or better than similar research in the literature.

In terms of orientation estimation, Vicon observations and Xsens measurements were presented to evaluate the effectiveness of proposed method. Since pitch angle changes most significantly than roll and heading in normal walking. Fig. 13 demon330 strates the foot pitch angle estimation during one step by proposed method, Vicon and Xsens respectively. It is observed that the proposed method produces a better result (errors no more than $3^{\circ}$ ) than the typical kalman filter method adopted by Xsens (errors 


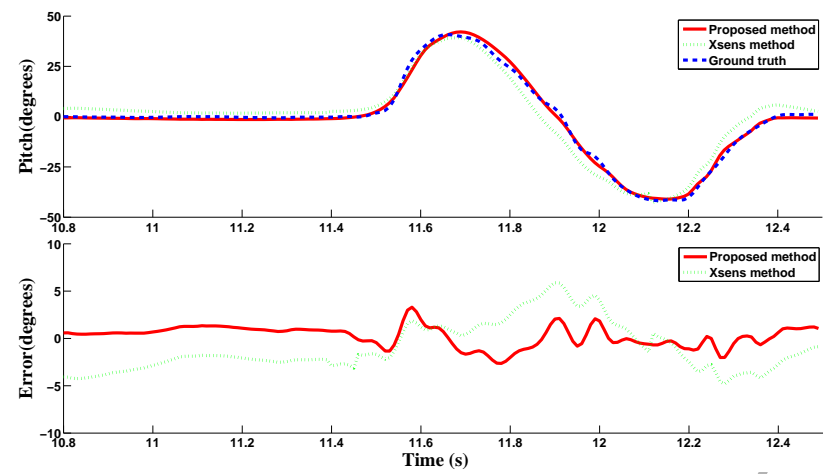

Figure 13. Foot pitch angle estimation of one step by proposed method, Xsens and Vicon system, as well as the difference errors of foot pitch angle estimation by proposed method and Xsens, respectively

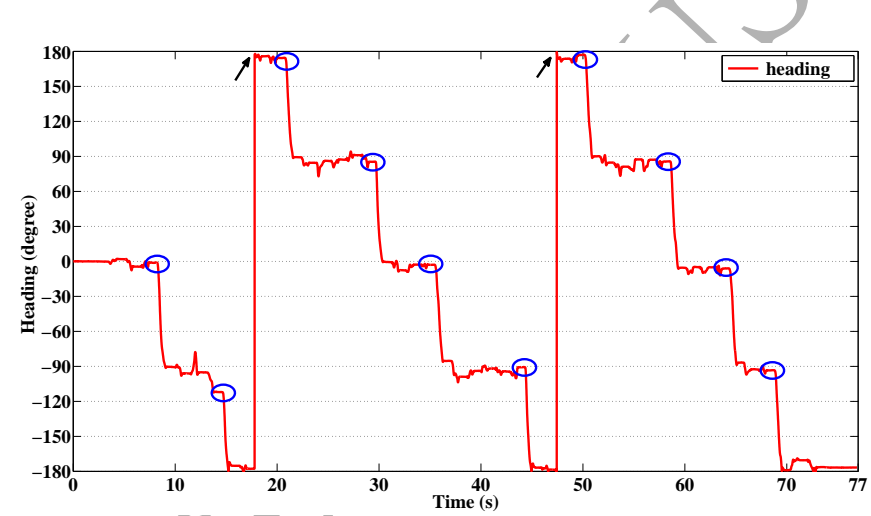

Figure 14. Heading change during rectangular level walking

exceed $5^{\circ}$ ), which tend to be more error prone due to to magnetic disturbance.

The change of heading angle during the rectangular walking trial is indicated in

Fig. 14. In this experiment, the subject followed the clockwise and turned right for ten times, i.e. the heading changed $10 \times\left(-90^{\circ}\right)$ relative to the navigation coordinate, ending up with a reverse heading $\left(-177.3^{\circ}\right)$ with respect to initial state. Note that there are some trip points when the heading is close to $\pm 180^{\circ}$, i.e., the heading could hop from $-180^{\circ}$ to $180^{\circ}$ due to the singularity character of Euler method. The definition of heading states that the value should be in the range of $\pm 180^{\circ}$, so we made some adjustments every time the subject walked at the reverse direction as indicated by black arrows in Fig. 14, ensuring that the heading angle falls into the set range. 
Table 3. INDOOR PDR RESULTS OF THE SAME SUBJECT FOR SIX TIMES

\begin{tabular}{cccccc}
\hline Trial Step count & Walking & Distance & Calculated & Number & Error \\
no. (true value) distance $\%$ error & heading & of $90^{\circ}$ & per turn
\end{tabular}

(m) $\quad\left(^{\circ}\right)$ turnings $\quad\left(^{\circ}\right)$

\begin{tabular}{lllllll}
\hline 1 & $57(57)$ & 49.51 & $0.39 \%$ & -176.4 & 10 & 0.4 \\
2 & $60(60)$ & 48.73 & $0.74 \%$ & 175.1 & 10 & 0.6 \\
3 & $59(59)$ & 49.19 & $0.17 \%$ & -172.9 & 10 & 0.7 \\
4 & $56(56)$ & 50.85 & $0.32 \%$ & -176.5 & 10 & 0.5 \\
5 & $55(55)$ & 51.33 & $0.26 \%$ & -173.2 & 10 & 0.7 \\
6 & $59(59)$ & 50.46 & $0.56 \%$ & 174.3 & 10 & 0.2 \\
\hline
\end{tabular}

\subsection{Outdoor experimental results}

We asked the subjects to circle the 400 meter track in an stadium, and the subjects were instructed to walk along the same specified track line (the fourth lane in this series of experiments, and the circumference is 423 meters). For comparison, we compared our PDR results of the outdoor walking trajectory with those obtained by complementary filter $(\mathrm{CF})$ in [28] and gradient descent algorithm (GDA) in [30]. Fig. 15 demonstrates the navigation trail using three methods for one lap walk around the athletic track overlaid onto Google map. We have conducted five outdoor trials of a specific subject and presented the results in Table 4 with measurement uncertainty. Note that the number of total walking steps is $\sim 321$ and the duration is $\sim 364$ seconds in these trials. The truth value of step number is provided by a pedometer. The corresponding nayigational trajectory are represented by different colors and line styles. The overall shape of the estimated trajectories seem very similar to the forth athletic track but the proposed EKF method shows improvement over those computed using other methods. Specifically, the trajectory for the proposed methods finished with an end-to-end error of $\sim 2.59$ meters, which is larger than CF method ( $\sim 1.96$ meters) but the overall 


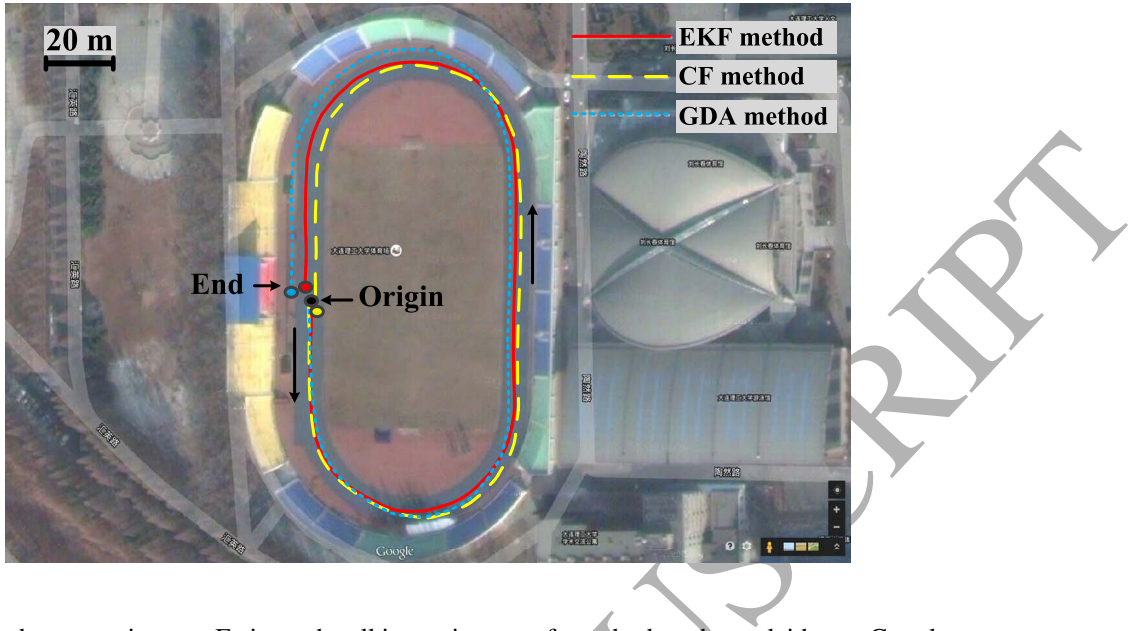

Figure 15. Outdoor experiments: Estimated walking trajectory of standard track overlaid onto Google map

Table 4. OUTDOOR PDR RESULTS USING THREE DIFFERENT METHODS

\begin{tabular}{cccccc}
\hline \hline Method & Step count & Walking & End-to-end & Error & Heading \\
& & distance $(\mathrm{m})$ & error $(\mathrm{m})$ & $\%$ & $\left({ }^{\circ}\right)$ \\
\hline \multirow{2}{*}{ EKF } & $321 \pm 6$ & $424.19 \pm 0.57$ & $2.59 \pm 0.012$ & 0.61 & $3.7 \pm 0.33$ \\
CF & $321 \pm 6$ & $423.83 \pm 0.81$ & $1.96 \pm 0.036$ & 0.46 & $7.1 \pm 0.49$ \\
GDA & $321 \pm 6$ & $424.72 \pm 0.49$ & $3.48 \pm 0.075$ & 0.82 & $-6.8 \pm 0.87$ \\
\hline \hline
\end{tabular}

trajectory is more consistent with the fourth track lane. Note that the biggest heading error occurs in the GDA method with the end heading angle of $\sim-6.8^{\circ}$, which is deviate from actual value. It can be concluded that the heading estimation error during the trajectory decreases the overall estimation quality.

As shown in Table 5, the overall error in distance is $\sim 0.3 \%$ of the total traveled distance, while the end-to-end (starting point to ending point) error is $\sim 0.65 \%$ of the total distance for the outdoor walking trial. The operation time for the two laps walking experiment is more than 10 minutes (687 seconds), demonstrating the drift reduction continue to work for a reasonable time. When comparing female and male 
Table 5. OUTDOOR PDR PERFORMANCE OF FOUR SUBJECTS

\begin{tabular}{ccccccc}
\hline \hline Subject & Gender & Step count & Walking & Error & End-to-end & Error \\
no. & & (true value) & distance $(\mathrm{m})$ & $\%(\mathrm{~W})$ & error $(\mathrm{m})$ & $\%(\mathrm{E})$ \\
\hline 1 & female & $321(321)$ & 424.23 & $0.29 \%$ & 2.74 & $0.64 \%$ \\
2 & female & $654(654)$ & 847.45 & $0.17 \%$ & 3.66 & $0.87 \%$ \\
3 & male & $297(297)$ & 424.53 & $0.36 \%$ & 2.79 & $0.66 \%$ \\
4 & male & $613(613)$ & 849.30 & $0.39 \%$ & 1.92 & $0.45 \%$ \\
\hline \hline
\end{tabular}

cohorts, male perform larger stride length and lower stride frequency than female. In conclusion, these experiments show the effectiveness of the proposed PDR approach. sistent with the study of [15]. The factors that influence the accuracy of orientation estimation may include magnetometer interference, sensor housing error and invalid specific domain assumptions, which may be revealed in further study.

In this paper, a PDR method based on inertial/magnetic sensors was proposed. The sensor system monitors the subjects walking information using the proposed pedestrian dead reckoning algorithm. Experimental results demonstrate that drift and error are well controlled by sensor fusion algorithm. Without pressure sensor, RFID tags 
and GPS, the self-contained inertial/magnetic sensor based method achieves an average distance error of $0.35 \%$ and the average end-to-end position error is around $0.65 \%$ of the total walked distance, taking account of various walking scenarios. It is difficult to directly compare the results with different experiments, because the algorithm performance largely depend on the sensor type, the ground surface and the trajectory, etc, Even though, our experimental results are comparable to or better than the literature:

1) Adopting the same inertial/magnetic sensor, Feliz et al. [33] proposed a gyroscope based distance estimation method with $7.81 \%$ error, the averaged distance error is $1.27 \%$ in [20] ; the position error of $0.4 \%$ was reported in the study of [29], which is quite accurate but lacks non-horizontal walking trials.

2) Adopting sensor of another vendor, the position accuracy of $0.3 \%$ reported in [24]; the $\Delta X Y$ radial distance error of $0.82 \%$ and the distance error of $0.27 \%$ reported in [28]; the distance error of $1.1 \%$ and the position error of $1.2 \%$ were reported in [14], the distance error of $0.43 \%$ for short distance walking and $4.31 \%$ for long distance indoor walking were reported in [34].

\section{CONCLUSION}

We have presented a multi-sensor fusion method to estimate the step length and foot orientation based on inertial/magnetic feet attached sensors. Contrary to similar works, a multisensor approach is applied in order to reduce uncertainty and to produce better estimations. Towards this end, a stance phase detection algorithm based on accelerometer and gyroscope measurements was presented, and an extended kalman filter based sensor fusion system is proposed. Initial results are encouraging, and the method has been proved effective for both short distance indoor walking trials and long distance outdoor pedestrian dead reckoning. We conclude that PDR could be served as a good supplement of other navigation approaches, or to extend navigation into areas where other navigation systems are unavailable.

Ongoing extended field experiments have been designed to validate and generalize the results for a heterogeneous populations and walking conditions. The performance of the proposed method could be further improved by taking into account the building 
structure and magnetic turbulence. Future research might aim to further improve the localization accuracy of the proposed method and conduct extensive trials across a large sample of participants and walking pattern, ensuring that the proposed PDR method is consistent across inter-subject. Another challenge is differentiating walking from running, and recognizing motions such as carrying heavy loads and taking electric lift. The method need to be enhanced to handle these various scenarios.

\section{Acknowledgment}

This work was supported in part by the National Natural Science Foundation of China under Grant 61473058 and Grant 61174027 , and in part by the Fundamen-

tal Research Funds for the Central Universities under Grant DUT16RC(3)081 and

DUT15ZD114. The authors would like to express their thanks to these funding bodies.

\section{References}

[1] E. Pulido Herrera, H. Kaufmann, J. Secue, R. Quirós, G. Fabregat, Improving data fusion in personal positioning systems for outdoor environments, Inf. Fusion 14 (1) (2013) 45-56

[2] A. Noureldin, A. El-Shafie, M. Bayoumi, GPS/INS integration utilizing dynamic neural networks for vehícular navigation, Inf. Fusion 12 (1) (2011) 48-57.

[3] H. Liu, H. Darabi, P. Banerjee, J. Liu, Survey of Wireless Indoor Positioning Techniques and Systems, IEEE Trans. Syst., Man, Cybern. C, Appl. Rev. 37 (6)

[4] Y. Li, Y. Zhuang, P. Zhang, H. Lan, X. Niu, N. El-Sheimy, An improved inertial/wifi/magnetic fusion structure for indoor navigation, Inf. Fusion 34 (2017) $101-119$.

[5] J. Xiao, S. L. Joseph, X. Zhang, B. Li, X. Li, J. Zhang, An Assistive Naviga440 tion Framework for the Visually Impaired, IEEE Trans. Hum. Mach. Syst. 45 (5) (2014) 1-6. 
[6] A. Millonig, K. Schechtner, Developing landmark-based pedestrian-navigation systems, IEEE Trans.Intell.Transp.Syst. 8 (1) (2007) 43-49.

[7] C. Huang, L. Lee, C. Ho, L. Wu, Z. Lai, Real-Time RFID Indoor Positioning System Based on Kalman-Filter Drift Removal and Heron-Bilateration Location Estimation, IEEE Trans. Instrum. Meas. 64 (3) (2015) 728-739.

[8] G. Fortino, R. Giannantonio, R. Gravina, P. Kuryloski, R. Jafari, Enabling Effective Programming and Flexible Management of Efficient Body Sensor Network Applications, IEEE Trans. Hum. Mach. Syst. 43 (1) (2013) 115-133.

[9] G. Fortino, S. Galzarano, R. Gravina, W. Li, A framework for collaborative computing and multi-sensor data fusion in body sensor networks, Inf. Fusion 22 (2015) 50-70.

[10] R. Gravina, P. Alinia, H. Ghasemzadeh, G. Fortino, Multi-Sensor Fusion in Body Sensor Networks: State-of-the-art and research challenges, Inf. Fusion 35 (2016) 68-80.

[11] N. Raveendranathan, S. Galzarano, V. Loseu, R. Gravina, R. Giannantonio, M. Sgroi, R. Jafari, G. Fortino, From modeling to implementation of virtual sensors in body sensor networks, IEEE Sensors J. 12 (3) (2012) 583-593.

[12] K. C. Lan, W. Y. Shih, Using smart-phones and floor plans for indoor location tracking, IEEE Trans. Hum. Mach. Syst. 44 (2) (2014) 211-221.

[13] B. Zhou, Q. Li, Q. Mao, W. Tu, Activity Sequence-Based Indoor Pedestrian Localization Using Smartphones, IEEE Trans. Hum. Mach. Syst. 45 (5) (2015) 113.

[14] S. Godha, G. Lachapelle, Foot mounted inertial system for pedestrian navigation, Meas. Sci. Technol. 19 (7) (2008) 1-9.

[15] O. Bebek, M. a. Suster, S. Rajgopal, M. J. Fu, X. Huang, M. C. Cavusoglu, D. J. Young, M. Mehregany, A. J. Van Den Bogert, C. H. Mastrangelo, Personal navigation via shoe mounted inertial measurement units, IEEE Trans. Instrum. Meas. 59 (11) (2010) 3018-3027. 
[16] S. K. Park, Y. S. Suh, A zero velocity detection algorithm using inertial sensors for pedestrian navigation systems, Sensors (Basel, Switzerland) 10 (10) (2010) 9163-9178.

[17] Widyawan, G. Pirkl, D. Munaretto, C. Fischer, C. L. An, P. Lukowicz, M. Klepal, A. Timm-Giel, J. Widmer, D. Pesch, H. Gellersen, Virtual lifeline: Multimodal sensor data fusion for robust navigation in unknown environments, Pervas. Mobile Comput. 8 (3) (2012) 388-401.

[18] R. M. Faragher, C. Sarno, M. Newman, Opportunistic radio SLAM for indoor navigation using smartphone sensors, IEEE Position Location and Navigation Symposium (2012) 120-128.

[19] J. Li, J. A. Besada, A. M. Bernardos, P. Tarrío, J. R. Casar, A novel system for object pose estimation using fused vision and inertial data, Inf. Fusion 33 (2017) $15-28$.

[20] A. R. Jiménez Ruiz, F. Seco Granja, J. C. Prieto Honorato, J. I. Guevara Rosas, Accurate pedestrian indoor navigation by tightly coupling foot-mounted IMU and RFID measurements, IEEE Trans. Instrum. Meas. 61 (1) (2012) 178-189.

[21] R. Harle, A Survey of Indoor Inertial Positioning Systems for Pedestrians, IEEE Commun. Sury. Tut. 15 (3) (2013) 1281-1293.

[22] F. Cavallo, a.M. Sabatini, V. Genovese, A step toward GPS/INS personal navigation systems: real-time assessment of gait by foot inertial sensing, IEEE/RSJ International Conference on Intelligent Robots and Systems (2005) 1187-1191.

[23] I. Skog, P. Händel, J.-O. Nilsson, J. Rantakokko, Zero-velocity detection — an algorithm evaluation., IEEE Trans. Biomed. Eng. 57 (11) (2010) 2657-2666.

[24] E. Foxlin, Pedestrian tracking with shoe-mounted inertial sensors, IEEE Comput. Graph. Appl. (2005) 38-46.

495 [25] Z. Wang, S. Qiu, Z. Cao, M. Jiang, Quantitative assessment of dual gait analysis based on inertial sensors with body sensor network, Sensor Rev. 33 (1) (2013) $48-56$. 
[26] S. Qiu, Z. Wang, H. Zhao, H. Hu, Using Distributed Wearable Sensors to Measure and Evaluate Human Lower Limb Motions, IEEE Trans. Instrum. Meas. 65 (4) (2016) 939-950.

[27] Y. S. Suh, A smoother for attitude and position estimation using inertial sensors with zero velocity intervals, IEEE Sensors J. 12 (5) (2012) 1255-1262.

[28] X. Yun, J. Calusdian, E. R. Bachmann, R. B. McGhee, Estimation of human foot motion during normal walking using inertial and magnetic sensor measurements, IEEE Trans. Instrum. Meas. 61 (7) (2012) 2059-2072.

[29] H. Fourati, Heterogeneous Data Fusion Algorithm for Pedestrian Navigation via Foot-Mounted Inertial Measurement Unit and Complementary Filter, IEEE Trans. Instrum. Meas. 64 (1) (2015) 221-229.

[30] S. O. H. Madgwick, A. J. L. Harrison, A. Vaidyanathan, Estimation of IMU and MARG orientation using a gradient descent algorithm, IEEE International Conference on Rehabilitation Robotics (2011) 1-7.

[31] K. Kunze, P. Lukowicz, Sensor Placement Variations in Wearable Activity Recognition, Pervas. Comput. 13 (4) (2014) 32-41.

[32] Z. Wang, D. Wu, R. Gravina, G. Fortino, Y. Jiang, K. Tang, Kernel fusion based extreme learning machine for cross-location activity recognition, Inf. Fusion 37 (2017) 1-9.

[33] R. Feliz, E. Zalama, J. G. Garcia-Bermejo, Pedestrian tracking using inertial sensors, J. Phys. Agents 3 (1) (2009) 35-43.

[34] X. Meng, Z. Q. Zhang, J. K. Wu, W. C. Wong, H. Yu, Self-contained pedestrian tracking during normal walking using an inertial/magnetic sensor module, IEEE Trans. Biomed. Eng. 61 (3) (2014) 892-899. 